\title{
Définition des volumes de besoin des rechargements massifs du littoral de Soulac-sur-Mer via l'utilisation d'un modèle Xbeach calibré avec des données topographiques mensuelles
}

\author{
Cédric OLCOMENDY ${ }^{1}$, Floriane BOGUN ${ }^{1}$, \\ Didier RIHOUEY ${ }^{1}$, Vincent MAZEIRAUX ${ }^{2}$
}

1. CASAGEC INGENIERIE, 18 rue Maryse Bastié, Zone de Maignon, 64600 Anglet, France.

olcomendy@casagec.fr; bogun@casagec.fr; rihouey@casagec.fr

2. Communauté de communes, Médoc Atlantique, 9 rue du Maréchal d'Ornano, 33780 Soulac-sur-Mer, France.

gemapi@ccmedocatlantique.fr

\section{Résumé :}

Le littoral sableux océanique nord-médocain, de la pointe de la Négade au sud (Soulacsur-Mer) à la jetée de Grave au nord (Verdon-sur-Mer), est sujet à des phénomènes d'érosion marine parmi les plus intenses des côtes françaises. Récemment, les tempêtes de l'hiver 2013-2014 ont très sévèrement touché ce littoral avec des reculs du trait de côte de près de $30 \mathrm{~m}$ sur certains secteurs, mettant en péril des zones urbanisées. Pour faire face à ces problèmes d'érosion chronique, la Communauté de Communes Médoc Atlantique (CDCMA) s'est dotée en 2018 d'une stratégie locale de gestion du phénomène d'érosion marine. L'un des axes de cette stratégie vise à restaurer le déficit chronique en sable des plages de Soulac-sur-Mer en rechargeant massivement le littoral à partir des sables présents dans l'estuaire externe de la Gironde. Par ailleurs, la CDCMA met en œuvre des suivis du trait de côte et des plages de Soulac-sur-Mer via des levés topographiques semestriels (estran et dune) depuis 2013.

Dans l'attente des rechargements massifs, un rechargement de $45000 \mathrm{~m}^{3}$ a été réalisé à l'automne 2018 et un suivi topographique mensuel a été mis en œuvre pour appréhender précisément la tenue des apports effectués en pied de dune. Ce suivi topographique est ici utilisé pour calibrer le modèle Xbeach (ROELVINK et al., 2009) sur ce pied de dune artificiel. Cette calibration spécifique permet de définir un coefficient de surrechargement 1,6. Ce résultat, couplé aux calculs de volumes perdus annuellement depuis 2013 sur les dunes de Soulac-sur-Mer, permet de définir les volumes de besoin pour stabiliser le recul du trait de côte des secteurs les plus sensibles.

Mots-clés :

Volumes de besoin, Coefficient de sur-rechargement, Modélisation Xbeach, Soulac-surMer 


\section{Thème 7 - Risques côtiers}

\section{Introduction}

Les rechargements sableux constituent une solution "douce" couramment utilisée pour atténuer les phénomènes d'érosion sur les littoraux sableux exposés. Au-delà de la faisabilité financière et technique d'une opération, le dimensionnement d'un projet de rechargement dépend principalement des objectifs fixés et de la dynamique morpho sédimentaire de la zone à protéger (HAMM et al., 2002).

Le littoral sableux océanique de Soulac-sur-Mer est sujet à des phénomènes d'érosion marine parmi les plus intenses des côtes françaises avec des valeurs mesurées pouvant atteindre $-7,5 \mathrm{~m} / \mathrm{an}$. Pour faire face à ces problèmes d'érosion chronique, la stratégie locale de gestion du trait de côte porté par la CDCMA (Communauté de Communes Médoc Atlantique) prévoit notamment des rechargements massifs du littoral dont les caractéristiques restent à définir.

La définition des volumes de besoin nécessaires pour stabiliser les plages fortement exposées à l'érosion constitue une problématique majeure pour les projets de rechargements. Si les guides d'ingénierie (USACE, 2002) considèrent qu'un coefficient de sur-rechargement $\mathrm{Ra}=1,05$ suffit si la granulométrie des sédiments d'apport est équivalente à celle des sédiments natifs, le retour d'expérience en Australie donne des coefficients de sur-rechargement de 1,1 à 2 (AECOM, 2010).

Les modèles numériques constituent des outils d'aide à la conception des projets de rechargements (CAPOBIANCO et al., 2002) et leur validation constitue un point clef de leur utilisation (KAMPHUIS, 2000). Le modèle XBeach (ROELVINK et al., 2009), qui profite d'un développement rapide et suscite actuellement l'engouement de la communauté scientifique (DESMAZES et al., 2015), permet de reproduire certains des phénomènes se déroulant pendant les tempêtes, et notamment l'érosion dunaire.

Dans cette étude, le modèle Xbeach a été calibré avec des données topographiques mensuelles réalisées à la suite d'un rechargement de $45000 \mathrm{~m}^{3}$ réalisé à l'automne 2018 et sur des secteurs non rechargés. Ces calibrations permettent de définir un coefficient de sur-rechargement spécifique. Ce résultat, couplé aux calculs de volumes perdus annuellement depuis 2013 sur les dunes de Soulac-sur-Mer, permet de définir les volumes de besoin pour stabiliser le recul du trait de côte des secteurs les plus sensibles.

\section{Site d'étude : Le littoral de Soulac-sur-Mer}

\subsection{Présentation générale du site}

Le littoral de Soulac-sur-Mer se localise au centre d'une province sédimentaire et d'une entité géomorphologique, comprises entre la Pointe de Grave au Nord et la Pointe de la Négade au Sud. Il constitue la ride Sud-Est de l'embouchure de la Gironde et s'intègre dans le système des passes de la Gironde (figure 1). La particularité de ce littoral se manifeste par une côte basse et sableuse (diamètre médian d'environ $400 \mu \mathrm{m}$ ). 


\section{XVI èmes Journées Nationales Génie Côtier - Génie Civil \\ Le Havre 2020}

Le stock sableux qui compose les plages du Nord-Médoc provient de différentes origines : les sables de la plaine des Landes, les sables marins du plateau continental et les sables fluviatiles actuels.

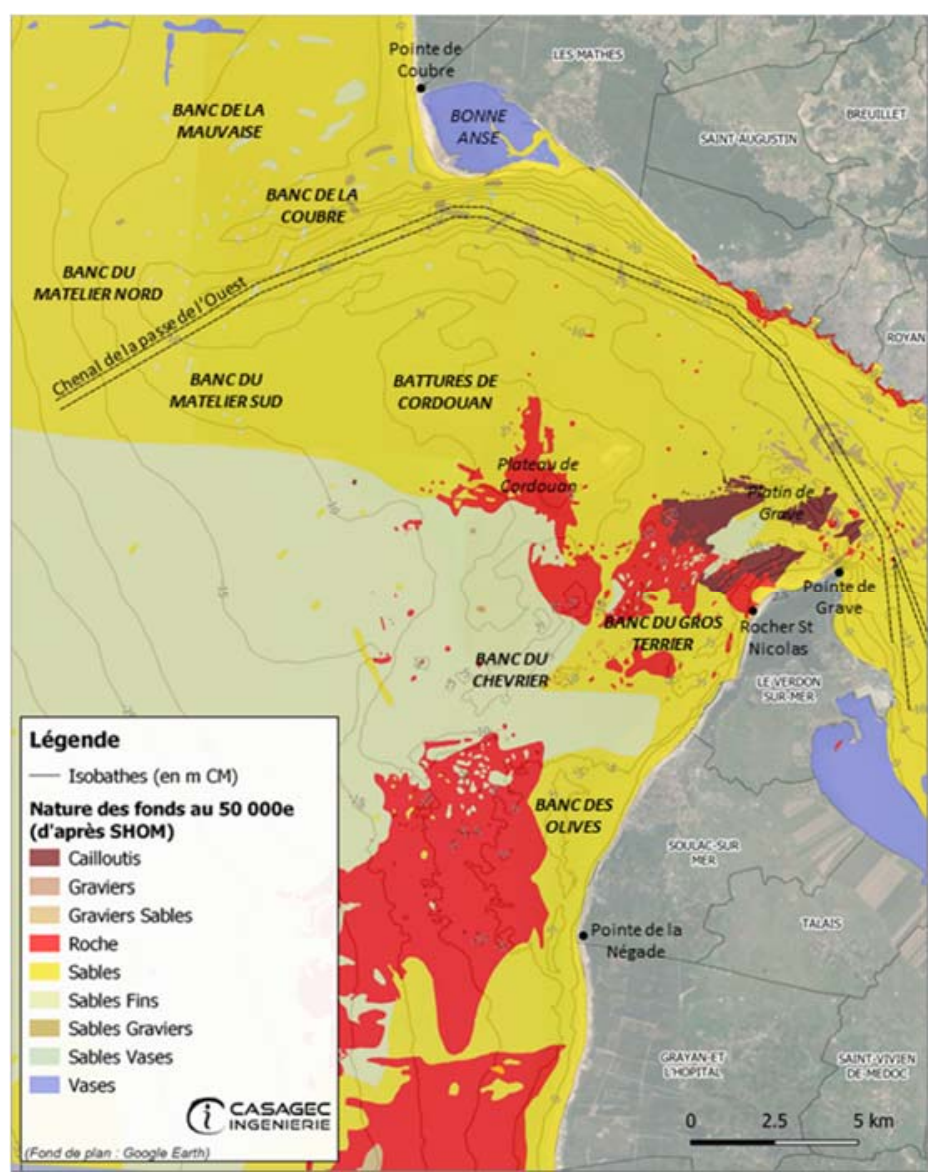

Figure 1. Localisation générale du littoral de Soulac-sur-Mer.

Sur le littoral, la plage est large de 200 à 300 mètres et est bordée par des formations dunaires de dimensions très variables (hauteurs pouvant aller de +10 à $+20 \mathrm{~m} \mathrm{NGF}$ ). Pendant les périodes de mer forte, les vagues entament le pied du cordon en formant des "microfalaises". L'étude se focalise sur les plages au Sud de Soulac-sur-Mer, entre les secteurs Sud Signal et Dunes de l'Amélie, secteur le plus sensible à l'érosion.

Sur le site d'étude, la marée est de type semi-diurne avec un marnage maximal de 5,4 m et un niveau moyen de 3,28 m CM. L'analyse statistique des données de houle de la bouée Oléron permet d'appréhender la climatologie moyenne des états de mer au large du site. Il en ressort que les directions principales de provenance sont de l'Ouest (N270) à 1 'Ouest-Nord-Ouest (N300) avec près de $91 \%$ des états de mer incidents. Les hauteurs significatives restent inférieures à $1,0 \mathrm{~m}$ pendant environ $31 \%$ du temps, inférieures à $2,0 \mathrm{~m}$ pendant environ $69 \%$, inférieures à $3,0 \mathrm{~m}$ pendant environ $87 \%$ du temps et peuvent atteindre 10,0 $\mathrm{m}$ lors des évènements les plus extrêmes. 


\section{Thème 7 - Risques côtiers}

\subsection{Evolutions récentes du littoral : entre 2013 et 2019}

La CDCMA s'est engagée dans une démarche de suivi des évolutions du littoral. Dans ce cadre, des levés topographiques saisonniers de la zone d'étude ont été réalisés entre 2013 et 2019. Ces acquisitions ont lieu deux fois par an (situations post-hivernale et postestivale) et comportent des levés topographiques le long de profils transverses espacés de $100 \mathrm{~m}$ qui s'étende du pied de dune jusqu'au bas de l'estran, ainsi qu'un levé scanner 3D pour la topographie de la face de dune (figure 2).

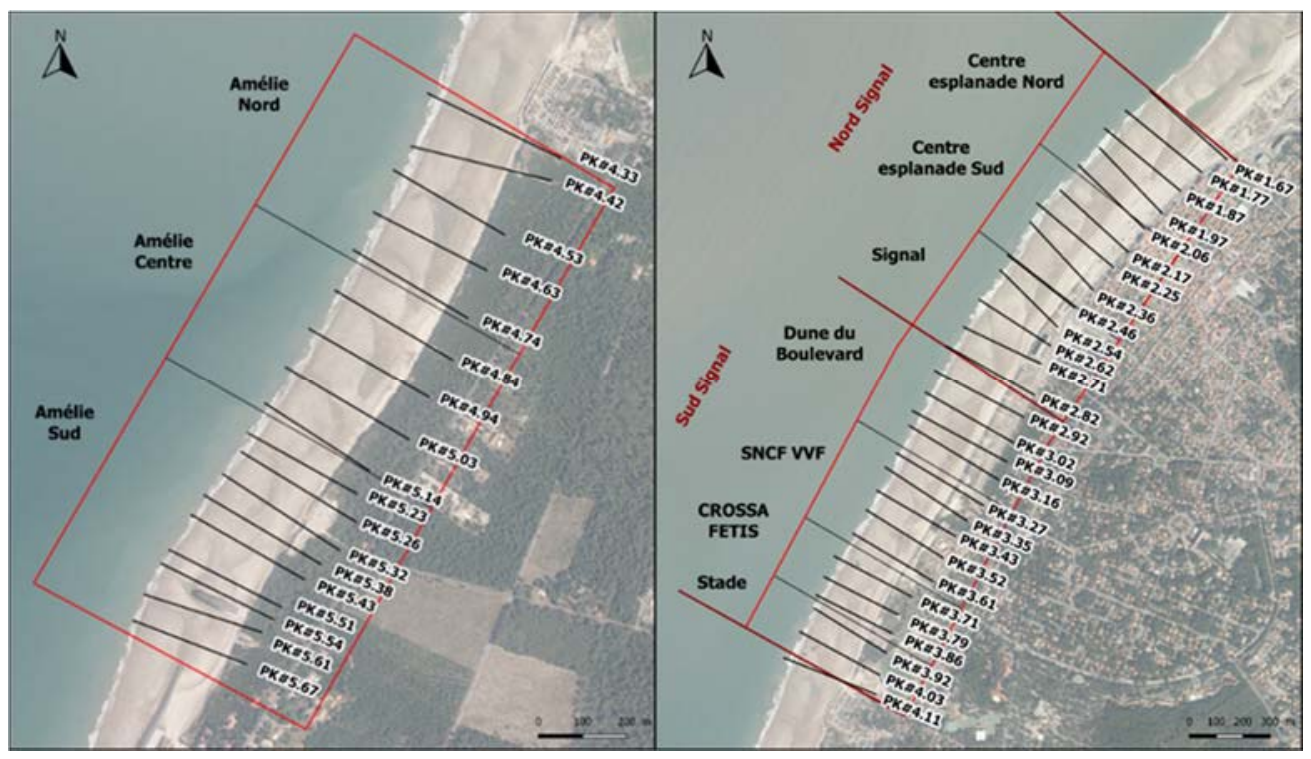

Figure 2. Localisation des levés topographiques.

Les données topographiques recueillies ont été analysées par le biais de deux indicateurs choisis pour quantifier les évolutions : la position du pied de dune définis par la rupture de pente entre le front dunaire et la plage, et les volumes de sables (perdus ou gagnés) sur le front dunaire entre deux levés successifs. Les résultats de l'analyse des données topographiques sont présentés dans le tableau 1. Ils font apparaitre des reculs du pied de dune entre 3,3 et 7,5 m/an et des pertes globales sur le secteur pour un hiver de $280000 \mathrm{~m}^{3}$ en moyenne et de $360000 \mathrm{~m}^{3}$ au maximum.

Tableau 1. Synthèse des données topographiques réalisées entre 2013 et 2019.

\begin{tabular}{|c|c|c|c|c|c|c|c|}
\hline & $\begin{array}{c}\text { Amélie } \\
\text { Sud }\end{array}$ & $\begin{array}{c}\text { Amélie } \\
\text { Centre }\end{array}$ & $\begin{array}{c}\text { Amélie } \\
\text { Nord }\end{array}$ & Stade & $\begin{array}{c}\text { CROSSA } \\
\text { FETIS }\end{array}$ & SNCF & Dune \\
\hline $\begin{array}{c}\text { Recul moyen du } \\
\text { pied de dune (m/an) }\end{array}$ & 3,3 & 5,3 & 6,6 & 5,9 & 7,5 & 4,6 & 4,4 \\
\hline $\begin{array}{c}\text { Pertes moyennes } \\
\text { par hiver (m }\end{array}$ & 48133 & 44011 & 52002 & 31536 & 27676 & 40853 & 32085 \\
\hline $\begin{array}{c}\text { Pertes maximales } \\
\text { pour un hiver (m }\end{array}$ & 72841 & 62440 & 61732 & 40698 & 28368 & 52495 & 41154 \\
\hline
\end{tabular}




\section{XVI'̀mes Journées Nationales Génie Côtier - Génie Civil \\ Le Havre 2020}

\section{Données et méthodes}

Un modèle Xbeach 1D a été implémenté sur plusieurs profils perpendiculaires à la côte (figure 3) afin d'évaluer la variabilité du recul dunaire entre des profils rechargés et des profils non rechargés. Le maillage est plus ou moins raffiné en fonction de la profondeur avec des tailles de maille comprises entre $30 \mathrm{~m}$ au large et $2 \mathrm{~m}$ au niveau de la plage. La topographie des profils est définie à partir des données disponibles : levé bathymétrique de juillet 2019 réalisé par la CDCMA et levés topographiques de 2018-2019.

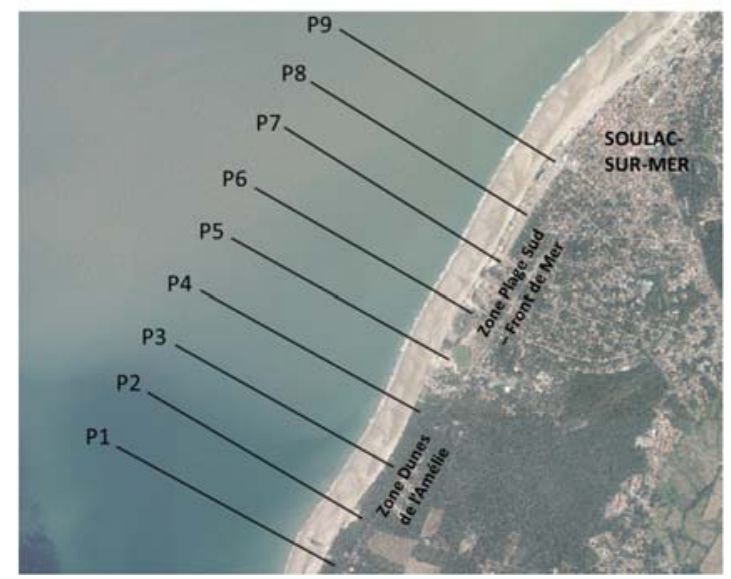

Figure 3. Localisation des profils de modélisation Xbeach.

Les conditions de houle et de niveau d'eau en entrée du modèle Xbeach ont été obtenues en reproduisant les états de mer de l'hiver 2018/2019 avec un modèle SWAN. Il s'agit d'un modèle grande emprise forcé par les données de houle de la bouée Oléron et par les niveaux d'eau du marégraphe de Port-Bloc. Afin de réduire les temps de calcul, seules les conditions susceptibles d'engendrer un recul du pied de dune (houles supérieures à 1 $\mathrm{m}$ et niveaux d'eau au-dessus de $4 \mathrm{~m} \mathrm{CM}$ ) ont été conservées dans la modélisation Xbeach (figure 4).

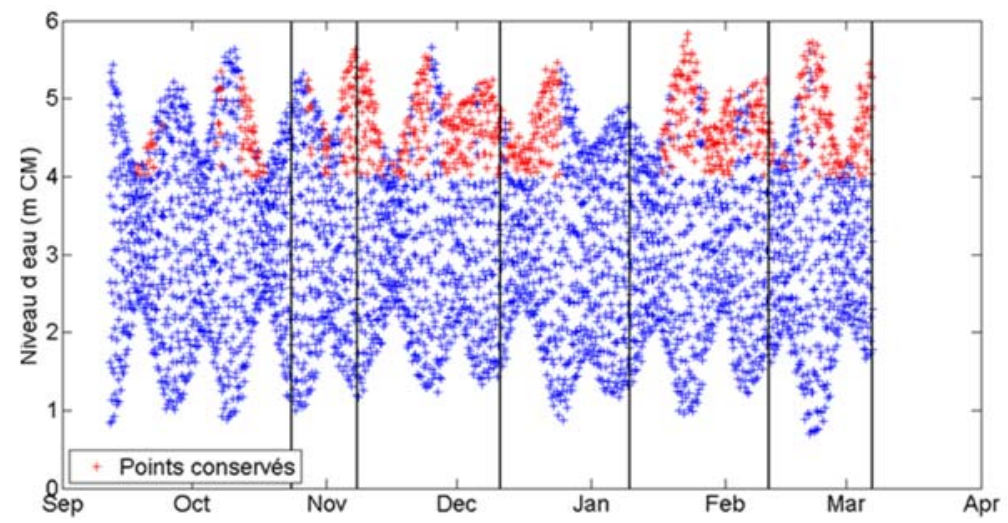

Figure 4. Dates conservées pour les conditions limites du modèle Xbeach. 


\section{Thème 7 - Risques côtiers}

Dans un premier temps, le modèle a été calibré sur un profil de plage rechargé ( $\mathrm{PK \# 4.03)}$ à l'aide du suivi topographique mensuel des rechargements mis en œuvre sur la plage des Naïades en Septembre 2018. La calibration a porté sur plusieurs paramètres du modèle : le facteur de calibration lié à l'asymétrie des vagues "facua", la pente critique d'écroulement de la dune "wetslp" et le coefficient de frottement "chezy". Cette calibration a permis d'obtenir un jeu de paramètres adapté à un profil de plage rechargé (facua $=0.2$, wetslp $=0.2$ et chezy=35) pour lequel l'érosion obtenue avec le modèle Xbeach correspond à celle observée grâce au suivi topographique (figure 5a-e).

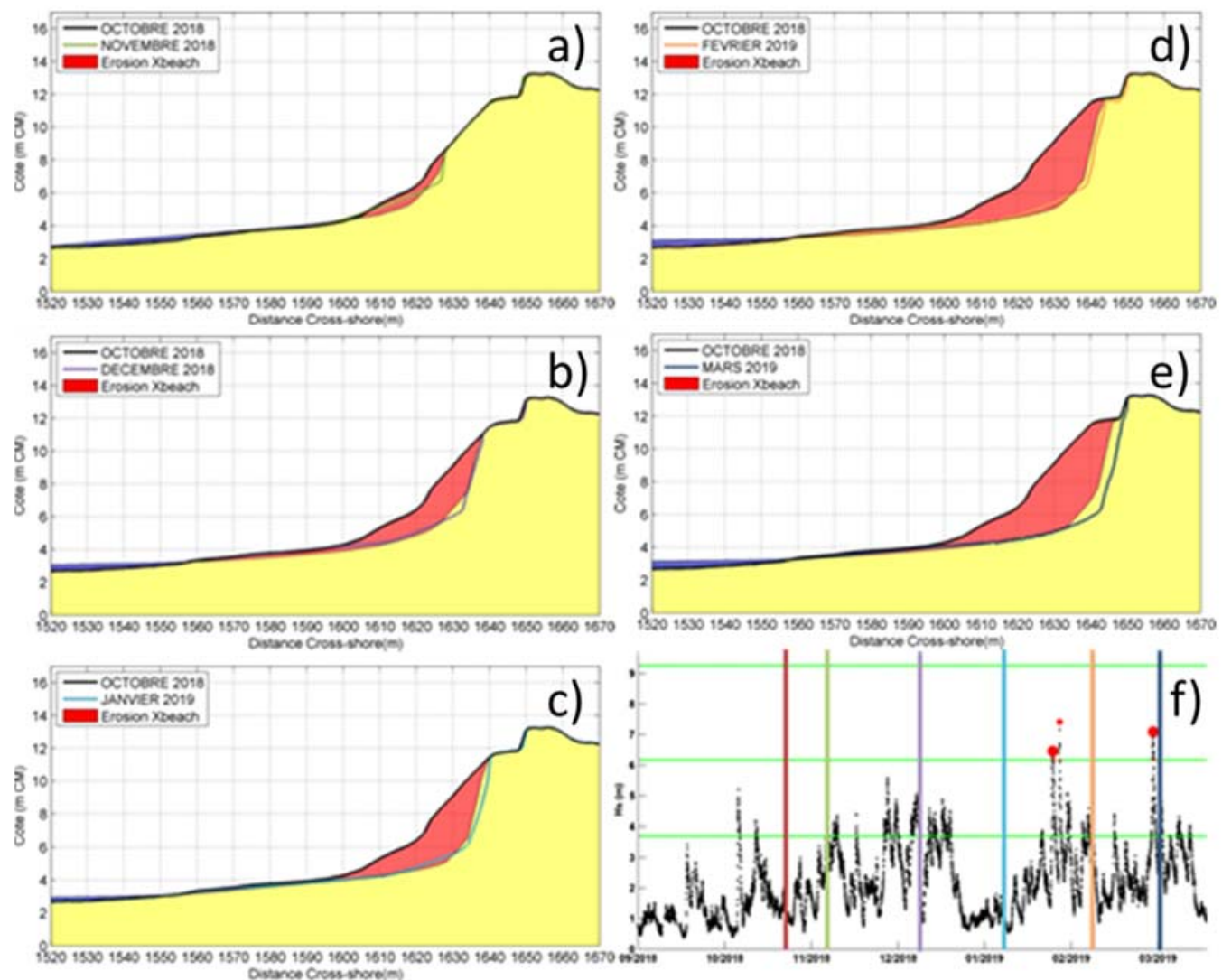

Figure 5. Calibration du modèle Xbeach sur un profil de plage rechargé (a,b,c,d,e) et série temporelle de la houle au large correspondante (f).

Dans un second temps, le modèle a été calibré sur un profil de plage sans rechargement (PK\#5.03) grâce aux données topographiques des levés saisonniers. L'érosion du profil de plage entre deux levés successifs (Novembre 2018 et Mai 2019) a été simulée avec Xbeach et comparée aux mesures. La figure 6 montre que l'érosion de la dune obtenue grâce à la simulation Xbeach est proche de celle observée sur les données du levé. Cette deuxième calibration a permis d'obtenir un jeu de paramètres (facua $=0.2$, wetslp $=0.35$ et chezy=35) adapté à un profil de plage non rechargé. 


\section{XVİ̀mes Journées Nationales Génie Côtier - Génie Civil \\ Le Havre 2020}

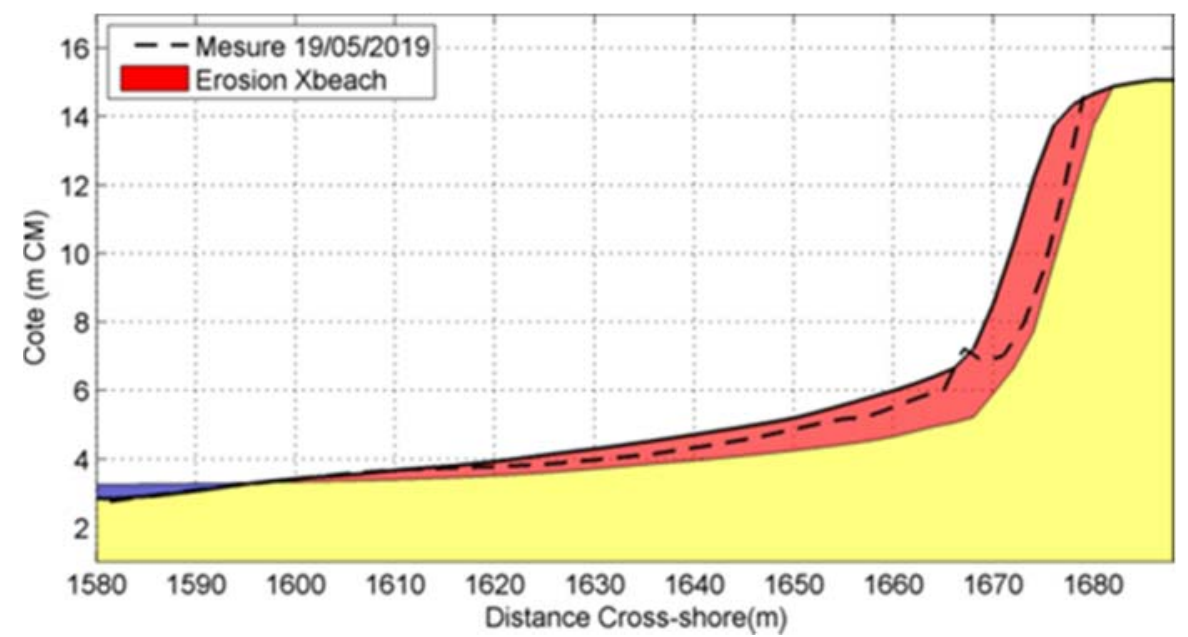

Figure 6. Calibration du modèle Xbeach sur un profil de plage non rechargé à partir des levés topographiques de Novembre 2018 et Mai 2019.

\section{Résultats}

Afin d'évaluer la variabilité du recul dunaire sur un profil de plage avec ou sans rechargement, l'hiver 2018/2019 a été simulé avec le modèle Xbeach sur un profil de plage en utilisant les deux calibrations définies précédemment. Le tableau 2 présente les volumes d'érosion obtenus par le modèle dans chacun des cas. Il apparait que l'érosion obtenue avec la calibration adaptée à un profil de plage rechargé (wetslp $=0,2)$ est plus importante que celle issue de la calibration pour un profil sans rechargement (wetslp = $0,35)$. Les volumes d'érosion sont environ 1,2 fois supérieurs sur l'ensemble de la plage et la dune, 1,6 fois supérieurs en considérant uniquement l'érosion de la dune.

Tableau 2. Volumes d'érosion pour l'hiver 2018/2019 d'après le modèle Xbeach.

\begin{tabular}{|c|c|c|c|}
\hline Volume d'érosion (m3) & $\begin{array}{c}\text { Calibration 1 } \\
\text { (profil rechargé) }\end{array}$ & $\begin{array}{c}\text { Calibration 2 } \\
\text { (profil non rechargé) }\end{array}$ & Ratio \\
\hline Plage et dune & 106,9 & 90,8 & 1,18 \\
\hline Dune uniquement & 67,3 & 42,3 & 1,59 \\
\hline
\end{tabular}

Les volumes nécessaires pour espérer stabiliser le trait de côte sur le secteur ont été calculés à partir des pertes hivernales issues de l'analyse des levés topographiques réalisés entre 2013 et 2019 (pertes moyennes par hiver de $280000 \mathrm{~m}^{3}$ et pertes maximales de $360000 \mathrm{~m}^{3}$ ). Ces pertes sont ensuite multipliées par le coefficient 1,6 obtenue avec le modèle Xbeach. In fine, les volumes de besoin annuels pour stabiliser 2,5 $\mathrm{km}$ du littoral de Soulac-sur-Mer sont estimés entre $448000 \mathrm{~m}^{3}$ (volume moyen) et $575000 \mathrm{~m}^{3}$ (volume maximal) de sable. Une valeur cible de $500000 \mathrm{~m}^{3} /$ an a été retenue pour la définition du programme de travaux soit $200 \mathrm{~m}^{3} / \mathrm{m} / \mathrm{an}$. Ces résultats corroborent les retours d'expériences (VAN RIJN, 2010 ; VAN RIJN, 2012) qui préconisent : de 10 à $50 \mathrm{~m}^{3} / \mathrm{m} / \mathrm{an}$ 


\section{Thème 7 - Risques côtiers}

pour un littoral faiblement exposé, de 50 à $150 \mathrm{~m}^{3} / \mathrm{m} /$ an pour un littoral moyennement exposé, de 150 à $300 \mathrm{~m}^{3} / \mathrm{m} /$ an pour un littoral fortement exposé.

\section{Conclusion et discussion}

Dans le cadre de cette étude, l'utilisation du code de calcul Xbeach, calibré à l'aide de suivis topographiques réguliers sur des secteurs rechargés et non rechargés, a permis de définir coefficient de sur-rechargement spécifique de 1,6. Ce résultat associé aux calculs de volumes perdus annuellement depuis 2013 sur les dunes de Soulac-sur-Mer, permet de retenir un volume de besoin de $500000 \mathrm{~m}^{3}$ /an pour stabiliser le recul du trait de côte des secteurs les plus sensibles. Xbeach a également été mis en œuvre pour tester la stabilité de différentes configurations de rechargement de pied de dune (largeur / hauteur).

\section{Références bibliographiques}

AECOM (2010). Beach sand nourishment scoping study maintaining Sydney's beach amenity against climate change sea level rise. Report, Sydney Coastal Councils Group. USACE- US Army Corps of Engineers (2002). Coastal Engineering Manual-CEM, Engineer Manual 1110-2-1100, Washington D.C., Volumes 1-6.

DESMAZES F., MULLER H., NICOLAE LERMA A., BULTEAU T., BALOUIN Y. (2015). Méthodologie de détermination et d'estimation du recul maximal des dunes lors d'évènements extrêmes. Phase 2 -Année 2. Rapport final, BRGM/RP-65439-FR, 94 p.

HAMM L., CAPOBIANCO M., DETTE H., LECHUGA A., SPANHOFF R., STIVE M. (2002). A summary of European experience with shore nourishment. Coast. Eng. 47, pp 237-264. https://doi.org/10.1016/S0378-3839(02)00127-8

KAMPHUIS J.W. (2000). Designing with models. $27^{\text {th }}$ International Conference on Coastal Engineering, Sydney, Australia, ASCE, New York, pp 19- 32.

CAPOBIANCO M., HANSON H., LARSON M., STEETZEL H., STIVE M.J.F., CHATELUS Y., AARNINKHOF S., KARAMBAS T. (2002). Nourishment design and evaluation: applicability of model concepts. Coast. Eng. 47(2), pp 113-135. https://doi.org/10.1016/S0378-3839(02)00123-0

ROELVINK D., RENIERS A., VAN DONGEREN A., VAN THIEL DE VRIES J., MCCALL., LESCINSKI J. (2009). Modelling storm impacts on beaches, dunes and barrier islands. Coastal Engineering, Volume 56 (11-12), pp 1133-1152. https://doi.org/10.1016/j.coastaleng.2009.08.006

VAN RIJN L.C. (2010). Coastal erosion control based on the concept of sediment cells. Eu-Project CONSCIENCE, Deltares, Delft, The Netherlands, www.conscience-eu.net.

VAN RIJN L.C. (2012). Principles of sedimentation and erosion engineering in rivers, estuaries and coastal seas. Aqua Publications, 580p. www.aquapublications.nl 